\title{
Rivaroxaban for Thromboprophylaxis in Acutely Ill Medical Patients
}

\author{
Alexander T. Cohen, M.D., Theodore E. Spiro, M.D., Harry R. Büller, M.D., \\ Lloyd Haskell, M.D., Dayi Hu, M.D., Russell Hull, M.B., B.S., \\ Alexandre Mebazaa, M.D., Geno Merli, M.D., Sebastian Schellong, M.D., \\ Alex C. Spyropoulos, M.D., and Victor Tapson, M.D., \\ for the MAGELLAN Investigators*
}

\section{A BSTRACT}

\section{BACKGROUND}

The clinically appropriate duration of thromboprophylaxis in hospitalized patients with acute medical illnesses is unknown. In this multicenter, randomized, doubleblind trial, we evaluated the efficacy and safety of oral rivaroxaban administered for an extended period, as compared with subcutaneous enoxaparin administered for a standard period, followed by placebo.

\section{METHODS}

We randomly assigned patients 40 years of age or older who were hospitalized for an acute medical illness to receive subcutaneous enoxaparin, $40 \mathrm{mg}$ once daily, for $10 \pm 4$ days and oral placebo for $35 \pm 4$ days or to receive subcutaneous placebo for $10 \pm 4$ days and oral rivaroxaban, $10 \mathrm{mg}$ once daily, for $35 \pm 4$ days. The primary efficacy outcomes were the composite of asymptomatic proximal or symptomatic venous thromboembolism up to day 10 (noninferiority test) and up to day 35 (superiority test). The principal safety outcome was the composite of major or clinically relevant nonmajor bleeding.

\section{RESULTS}

A total of 8101 patients underwent randomization. A primary efficacy outcome event occurred in 78 of 2938 patients (2.7\%) receiving rivaroxaban and 82 of 2993 patients (2.7\%) receiving enoxaparin at day 10 (relative risk with rivaroxaban, 0.97; 95\% confidence interval [CI], 0.71 to $1.31 ; \mathrm{P}=0.003$ for noninferiority) and in 131 of 2967 patients (4.4\%) who received rivaroxaban and 175 of 3057 patients (5.7\%) who received enoxaparin followed by placebo at day 35 (relative risk, 0.77 ; 95\% CI, 0.62 to 0.96; $\mathrm{P}=0.02$ ). A principal safety outcome event occurred in 111 of 3997 patients $(2.8 \%)$ in the rivaroxaban group and 49 of 4001 patients $(1.2 \%)$ in the enoxaparin group at day $10(\mathrm{P}<0.001)$ and in 164 patients $(4.1 \%)$ and 67 patients $(1.7 \%)$ in the respective groups at day $35(\mathrm{P}<0.001)$.

\section{CONCLUSIONS}

In acutely ill medical patients, rivaroxaban was noninferior to enoxaparin for standard-duration thromboprophylaxis. Extended-duration rivaroxaban reduced the risk of venous thromboembolism. Rivaroxaban was associated with an increased risk of bleeding. (Funded by Bayer HealthCare Pharmaceuticals and Janssen Research and Development; MAGELLAN ClinicalTrials.gov number, NCT00571649.)

From King's College Hospital, London (A.T.C.); Bayer HealthCare Pharmaceuticals, Montville (T.E.S.), and Janssen Research and Development, Raritan (L.H.) — both in New Jersey; Academic Medical Center, Amsterdam (H.R.B.); People's Hospital of Peking University, Beijing (D.H.); Foothills Hospital, Calgary, AB, Canada (R.H.); University Paris Diderot, pôle de recherche et d'enseignement supérieur Sorbonne Paris Cite and Hôpital Lariboisière, Paris (A.M.); Thomas Jefferson Medical Center, Philadelphia (G.M.); Dresden-Friedrichstadt Hospital, Dresden, Germany (S.S.); Hofstra North ShoreLong Island Jewish School of Medicine, Manhasset, NY (A.C.S.); and Duke University Medical Center, Durham, NC (V.T.). Address reprint requests to Dr. Cohen at Vascular Medicine, Department of Vascular Surgery, King's College Hospital, London SE5 9RS, United Kingdom, or at alexander.cohen@kcl.ac.uk.

\section{*The investigators participating in the Multicenter, Randomized, Parallel Group Efficacy and Safety Study for the Prevention of Venous Thromboembo- lism in Hospitalized Acutely III Medical Patients Comparing Rivaroxaban with Enoxaparin (MAGELLAN) and the study committees are listed in the Sup- plementary Appendix, available at NEJM.org.}

N Engl J Med 2013;368:513-23. DOI: 10.1056/NEJMoal111096 Copyright (๑) 2013 Massachusetts Medical Society. 
P ATIENTS WITH ACTIVE CANCER, STROKE, myocardial infarction, or acute exacerbations of a variety of medical conditions are at increased risk for venous thromboembolism. ${ }^{1}$ Prolonged immobilization and risk factors such as an age older than 75 years, chronic heart failure, a history of venous thromboembolism, and obesity can increase this risk further. ${ }^{2,3}$

Randomized, controlled trials involving hospitalized patients at increased risk for venous thromboembolism have shown the benefits of administering anticoagulant agents for up to 14 days, ${ }^{4-8}$ and guidelines recommend the use of unfractionated heparin, low-molecular-weight heparins, or fondaparinux in such patients. ${ }^{9}$ There is some evidence that the risk of venous thromboembolism in acutely ill medical patients persists after hospital discharge ${ }^{10}$; however, no studies have supported the routine use of extended thromboprophylaxis. ${ }^{11,12}$

Rivaroxaban is an oral, direct factor $\mathrm{Xa}$ inhibitor that is used for the prevention of venous thromboembolism after elective hip-replacement or knee-replacement surgery in adults. The Multicenter, Randomized, Parallel Group Efficacy and Safety Study for the Prevention of Venous Thromboembolism in Hospitalized Acutely Ill Medical Patients Comparing Rivaroxaban with Enoxaparin (MAGELLAN) was designed to assess the efficacy and safety of rivaroxaban administered for 35 days, as compared with enoxaparin administered for 10 days and followed by placebo, in a heterogeneous population of patients 40 years of age or older with reduced mobility and an acute medical illness requiring hospitalization.

\section{METHODS}

\section{STUDY OVERSIGHT}

MAGELLAN was a randomized, double-blind, active-comparator-controlled, multinational clinical trial. ${ }^{13}$ The study was designed and supervised by the steering committee (see the Supplementary Appendix, available with the full text of this article at NEJM.org) and was sponsored by Bayer HealthCare Pharmaceuticals and Janssen Research and Development. The members of the steering committee signed confidentiality agreements with the study sponsors. The data were collected and analyzed by the sponsors. All the authors had full access to the data and analyses and contributed to the writing of the manuscript. Editorial assistance was provided by Chameleon Com- munications. The steering committee made the decision to submit the manuscript for publication. The academic authors vouch for the accuracy and completeness of the report and for the fidelity of the report to the trial protocol, which is available at NEJM.org.

The trial was conducted in accordance with the Declaration of Helsinki and local regulations. The protocol was approved by the relevant local institutional review boards and ethics committees, and written informed consent was obtained from each patient before any study-specific procedures were performed.

\section{PATIENTS, TREATMENTS, AND FOLLOW-UP}

Patients were eligible for the study if they were 40 years of age or older, had been hospitalized for a specified acute medical illness for less than 72 hours before randomization, and had reduced mobility. Full inclusion and exclusion criteria are provided in Table S1 in the Supplementary Appendix. Randomization was performed in permuted blocks with the use of an interactive voiceresponse system, with stratification according to center. Eligible patients were randomly assigned to receive subcutaneous enoxaparin, $40 \mathrm{mg}$ once daily, for $10 \pm 4$ days and oral placebo, once daily, for $35 \pm 4$ days or to receive subcutaneous placebo, once daily, for $10 \pm 4$ days and oral rivaroxaban, $10 \mathrm{mg}$ once daily, for $35 \pm 4$ days.

The protocol called for ultrasonography to be performed in all patients for the detection of asymptomatic deep-vein thrombosis after the last dose of study medication or matching placebo was administered on day $10 \pm 4$ and on day $35 \pm 4$, as described previously. ${ }^{13}$ During the followup period, clinically suspected cases of deepvein thrombosis were confirmed with the use of ultrasonography or other vascular imaging techniques, and clinically suspected pulmonary embolism was confirmed with the use of thoracic spiral computed tomography, ventilation-perfusion lung scanning with chest radiography, or pulmonary angiography.

\section{OUTCOME MEASURES}

All outcomes were assessed by an independent, central adjudication committee whose members were unaware of the study assignments. There were two coprimary efficacy outcomes. The first was a composite of asymptomatic proximal deepvein thrombosis, symptomatic proximal or distal deep-vein thrombosis, symptomatic nonfatal pul- 
monary embolism, or death related to venous thromboembolism from day 1 to day 10 (day 10 analysis). The second was this same composite outcome from day 1 to day 35 (day 35 analysis). The primary day 10 analysis was prespecified to be a noninferiority analysis, and the primary day 35 analysis was prespecified to be a superiority analysis.

There were two major secondary efficacy outcomes: a composite of asymptomatic proximal deep-vein thrombosis, symptomatic proximal or distal deep-vein thrombosis, symptomatic nonfatal pulmonary embolism, or death from any cause (i.e., the primary outcome, with the component of death related to venous thromboembolism replaced by death from any cause), up to day 35; and a superiority analysis of the primary efficacy outcome up to day 10 in the modified intention-to-treat population. Other secondary efficacy outcomes included the incidence of symptomatic venous thromboembolism up to day 10 and the incidence up to day 35; the incidence of each of the components of the two coprimary efficacy outcomes; and the incidence of the composite of cardiovascular death, acute myocardial infarction, or acute ischemic stroke up to day 10 and up to day 35 .

The principal safety outcome was clinically relevant bleeding, which was a composite of major bleeding or clinically relevant nonmajor bleeding events observed no later than 2 days after administration of the last dose of doubleblind study medication. The definitions of major bleeding and clinically relevant nonmajor bleeding are provided in Table S2 in the Supplementary Appendix. Overt bleeding episodes that did not meet the criteria for either of these outcomes, adverse events that occurred during the treatment period, and abnormal laboratory results were also evaluated. The outcome of net clinical benefit or harm was assessed as the composite of the primary efficacy outcome or the principal safety outcome up to day 10 and up to day 35 .

\section{STATISTICAL ANALYSIS}

Details of the statistical analyses have been reported previously. ${ }^{13}$ In brief, two populations were defined - a modified intention-to-treat population and a per-protocol population - both for the noninferiority (day 10) efficacy analysis and for the superiority (day 35) efficacy analysis. For both efficacy analyses, patients were included in the modified intention-to-treat population if they met the study inclusion criteria, had received at least one dose of study medication, and had an adequate assessment of venous thromboembolism. Patients were included in the per-protocol population if they met the criteria for inclusion in the modified intention-to-treat population, underwent an adequate assessment of venous thromboembolism not later than 2 days after administration of the last dose of study drug, and had no major protocol violations. Patients were included in the safety population if they had received at least one dose of study medication.

For both coprimary efficacy outcomes, the relative risk ratio of the incidence rates (rivaroxaban as compared with enoxaparin) was estimated on the basis of a Mantel-Haenszel model with stratification according to geographic region. The perprotocol population was the primary population for the noninferiority analysis (at day 10), and the modified intention-to-treat population was the primary population for the superiority analysis (at day 35). All statistical tests were one-sided, with a type I error rate of $2.5 \%$, and two-sided $95 \%$ confidence intervals were calculated. ${ }^{14}$

We estimated that with a sample of 2876 patients per group who could be evaluated for the coprimary efficacy outcomes, the study would have at least $90 \%$ power to determine both the noninferiority (for the day 10 analysis) and superiority (for the day 35 analysis) of rivaroxaban as compared with enoxaparin. For the day 10 noninferiority analysis, the following assumptions were made: a $2.2 \%$ event rate in the enoxaparin group, a $1.4 \%$ event rate in the rivaroxaban group, and a relative risk reduction with rivaroxaban of at least $35 \%$. The estimate of the sample size assumed a relative risk noninferiority margin of 1.5. For the day 35 superiority analysis, the following assumptions were made: a $4.0 \%$ event rate in the enoxaparin group, a $2.4 \%$ event rate in the rivaroxaban group, and a relative risk reduction with rivaroxaban of at least $40 \%$.

For the safety analysis, all statistical tests were two-sided, with a type I error rate of 5\%. The outcomes of the composite of major bleeding or clinically relevant nonmajor bleeding and of major bleeding alone were analyzed in the same way as were the coprimary efficacy outcomes. All other safety data were analyzed descriptively. In exploratory analyses, the incidence rates, as well as the relative risk and the corresponding nonstratified asymptotic two-sided $95 \%$ confidence intervals, of the primary efficacy and principal 
safety outcomes in each treatment group were reported in subgroups defined according to age, sex, and acute medical condition.

RESULTS

\section{STUDY POPULATIONS}

From December 2007 through July 2010, a total of 8428 patients were enrolled at 556 sites in 52 countries. Of these patients, 8101 underwent randomization: 4050 to the rivaroxaban group and 4051 to the enoxaparin group (Fig. 1). The baseline characteristics of the patients, the incidences of acute medical conditions, and the risk factors for venous thromboembolism were well balanced between the two groups (Table 1). More than 30\% of the patients overall had two or more acute medical conditions, and the median duration of hospitalization in both groups was 11 days.

The numbers of patients included in each analysis are shown in Figure 1. A total of 53 patients in the rivaroxaban group and 50 patients in the enoxaparin group did not receive the study medication and were excluded from the safety analyses. In the rivaroxaban group, an additional 765 patients were excluded from the modified intention-to-treat analysis at day 10 and 1030 at day 35, with the lack of an adequate assessment of venous thromboembolism as the main reason for exclusion. In the enoxaparin group, an additional 730 patients were excluded from the modified intention-to-treat analysis at day 10 and 944 at day 35. A comparison of the baseline characteristics of patients who underwent venous ultrasonography at day 35 and those who did not is shown in Table S3 in the Supplementary Appendix. An additional 294 patients in the rivaroxaban group and 278 in the enoxaparin group were excluded from the per-protocol analysis. The total numbers of patients included in each of the analysis populations are provided in Table S4 in the Supplementary Appendix.

\section{EFFICACY OUTCOMES}

In the day 10 analysis of data from the per-protocol population, 78 of 2938 patients (2.7\%) in the rivaroxaban group and 82 of 2993 patients (2.7\%) in the enoxaparin group had a primary outcome event, with rivaroxaban meeting the prespecified criterion for noninferiority (relative risk with rivaroxaban, $0.97 ; 95 \%$ confidence interval [CI], 0.71 to $1.31 ; \mathrm{P}=0.003$ for noninferiority) (Table 2). In
Figure 1 (facing page). Randomization, Treatment, and Inclusion in Analyses.

Some patients met more than one exclusion criterion. VTE denotes venous thromboembolism.

the day 35 analysis of data from the modified intention-to-treat population, 131 of $2967 \mathrm{pa}$ tients $(4.4 \%)$ in the group that received extendedduration rivaroxaban, as compared with 175 of 3057 patients $(5.7 \%)$ in the group that received enoxaparin followed by placebo, had a primary outcome event; thus, extended-duration rivaroxaban met the conventional criterion for superiority (relative risk with rivaroxaban, 0.77; 95\% CI, 0.62 to $0.96 ; \mathrm{P}=0.02$ ) (Table 2). Primary efficacy outcome events between day 11 and day 35 occurred in 72 of 2934 patients (2.5\%) who received rivaroxaban and in 114 of 3017 patients (3.8\%) who received enoxaparin followed by placebo (relative risk with rivaroxaban, 0.65 ; $95 \% \mathrm{CI}, 0.49$ to 0.87 ; $\mathrm{P}=0.004)$. There was no evidence of heterogeneity according to subgroup with respect to the primary outcome at day 35 (Fig. S1 in the Supplementary Appendix).

With respect to the first major secondary efficacy outcome (the primary efficacy outcome up to day 35 , with death related to venous thromboembolism replaced by death from any cause), an event occurred in 266 of 3096 patients (8.6\%) in the group that received extended-duration rivaroxaban and in 293 of 3169 patients (9.2\%) in the group that received enoxaparin followed by placebo (relative risk with rivaroxaban, 0.93; 95\% CI, 0.80 to 1.09; $\mathrm{P}=0.38$ ) (Table 3). With respect to the second major secondary efficacy outcome (the primary efficacy outcome up to day 10 for a superiority analysis in the modified intention-totreat population), an event occurred in 98 of 3232 patients $(3.0 \%)$ in the rivaroxaban group and in 100 of 3271 patients (3.1\%) in the enoxaparin group (relative risk with rivaroxaban, 0.99; $95 \%$ CI, 0.75 to $1.30 ; \mathrm{P}=0.95$ ). The incidences of symptomatic venous thromboembolism and of the composite of cardiovascular death, acute myocardial infarction, or acute ischemic stroke did not differ significantly between the two groups at day 10 or at day 35 .

\section{SAFETY OUTCOMES}

Between day 1 and day 10, an episode of clinically relevant bleeding occurred in 111 of 3997 


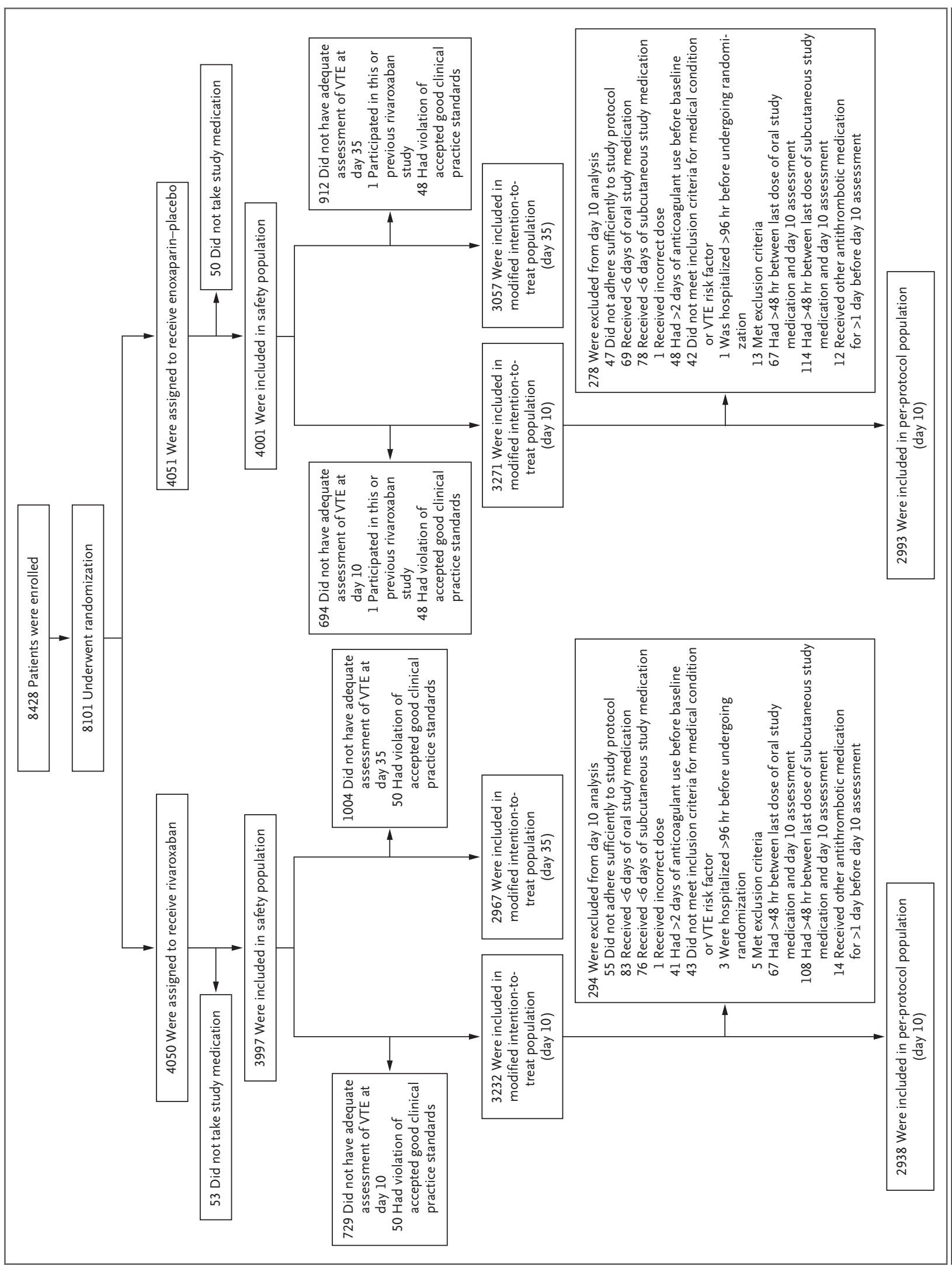

N ENGLJ MED 368;6 NEJM.ORG FEBRUARY 7, 2013

The New England Journal of Medicine 


\begin{tabular}{|c|c|c|}
\hline Characteristic & Rivaroxaban $(\mathrm{N}=4050)$ & Enoxaparin $(\mathrm{N}=4051)$ \\
\hline Median age $-\mathrm{yr}$ & 71.0 & 71.0 \\
\hline Male sex — no. (\%) & $2253(55.6)$ & $2136(52.7)$ \\
\hline Mean weight - kg & 77.5 & 77.3 \\
\hline Mean body-mass index $†$ & 28.2 & 28.2 \\
\hline Median duration of hospitalization - days & 11.0 & 11.0 \\
\hline \multicolumn{3}{|l|}{ Creatinine clearance - no. $(\%) \ddagger$} \\
\hline$<30 \mathrm{ml} / \mathrm{min}$ & $82(2.0)$ & $64(1.6)$ \\
\hline 30 to $<50 \mathrm{ml} / \mathrm{min}$ & $788(19.5)$ & 807 (19.9) \\
\hline 50 to $\leq 80 \mathrm{ml} / \mathrm{min}$ & $1500(37.0)$ & $1546(38.2)$ \\
\hline$>80 \mathrm{ml} / \mathrm{min}$ & $1582(39.1)$ & $1537(37.9)$ \\
\hline \multicolumn{3}{|l|}{ Race - no. (\%)』 } \\
\hline White & $2784(68.7)$ & $2744(67.7)$ \\
\hline Asian & 804 (19.9) & $806(19.9)$ \\
\hline Other & $280(6.9)$ & $290(7.2)$ \\
\hline Median D-dimer level $-\mu \mathrm{g} / \mathrm{m} \mid \boldsymbol{q}$ & 0.94 & 0.95 \\
\hline \multicolumn{3}{|l|}{ Acute medical condition — no. (\%) } \\
\hline Infectious disease & $1854(45.8)$ & $1828(45.1)$ \\
\hline Heart failure & $1308(32.3)$ & $1312(32.4)$ \\
\hline Respiratory insufficiency & $1105(27.3)$ & $1163(28.7)$ \\
\hline Ischemic stroke & $699(17.3)$ & $700(17.3)$ \\
\hline Active cancer & $296(7.3)$ & $296(7.3)$ \\
\hline Inflammatory or rheumatic disease & $152(3.8)$ & $151(3.7)$ \\
\hline Other & $34(0.8)$ & $24(0.6)$ \\
\hline$\geq 2$ Medical conditions & $1240(30.6)$ & $1270(31.4)$ \\
\hline \multicolumn{3}{|l|}{ Risk factor for VTE — no. (\%) } \\
\hline Age $\geq 75 \mathrm{yr}$ & $1551(38.3)$ & $1565(38.6)$ \\
\hline History of heart failure $\|$ & $1408(34.8)$ & $1382(34.1)$ \\
\hline History of cancer & $700(17.3)$ & $678(16.7)$ \\
\hline Acute ischemic stroke with leg paresis & $661(16.3)$ & $668(16.5)$ \\
\hline Chronic venous insufficiency & $617(15.2)$ & $579(14.3)$ \\
\hline Body-mass index $\geq 35$ & $612(15.1)$ & $618(15.3)$ \\
\hline Acute infectious disease & $566(14.0)$ & $601(14.8)$ \\
\hline Severe varicosis & $501(12.4)$ & $461(11.4)$ \\
\hline History of DVT or pulmonary embolism & $202(5.0)$ & $179(4.4)$ \\
\hline Hormone-replacement therapy & $48(1.2)$ & $50(1.2)$ \\
\hline Major surgery within the previous 6 to $12 \mathrm{wk}$ & $29(0.7)$ & $32(0.8)$ \\
\hline Hereditary or acquired thrombophilia & $15(0.4)$ & $9(0.2)$ \\
\hline Serious trauma within the previous 6 to $12 \mathrm{wk}$ & $7(0.2)$ & $7(0.2)$ \\
\hline
\end{tabular}

* There were no significant differences between the two groups in any of the baseline characteristics listed here. DVT denotes deep-vein thrombosis, and VTE venous thromboembolism.

$\uparrow$ The body-mass index is the weight in kilograms divided by the square of the height in meters.

$\checkmark$ Creatinine clearance levels were calculated with the use of the equation of Cockcroft and Gault. The values shown are day 1 levels.

$\int$ Race was self-reported.

9 The D-dimer level was calculated in the safety population (all patients who underwent randomization and received at least one dose of study medication), which included 7998 patients (3997 in the rivaroxaban group and 4001 in the enoxaparin group). The normal range is less than $0.5 \mu \mathrm{g}$ per milliliter.

|| Included are patients with a history of New York Heart Association heart failure class III or IV. 


\begin{tabular}{|c|c|c|c|c|c|c|c|c|}
\hline \multirow[t]{3}{*}{ Outcome } & \multicolumn{4}{|c|}{ Day 10} & \multicolumn{4}{|c|}{ Day 35} \\
\hline & $\begin{array}{l}\text { Rivaroxaban } \\
(N=2938)\end{array}$ & $\begin{array}{c}\text { Enoxaparin } \\
(\mathrm{N}=2993)\end{array}$ & $\begin{array}{c}\text { Relative Risk } \\
(95 \% \mathrm{Cl}) *\end{array}$ & P Value $†$ & $\begin{array}{c}\text { Rivaroxaban } \\
(\mathrm{N}=2967)\end{array}$ & $\begin{array}{c}\text { Enoxaparin- } \\
\text { Placebo } \\
(\mathrm{N}=3057)\end{array}$ & $\begin{array}{c}\text { Relative Risk } \\
(95 \% \mathrm{Cl})^{*}\end{array}$ & P Value \\
\hline & \multicolumn{2}{|c|}{ no. (\%) } & \multicolumn{6}{|c|}{ no. (\%) } \\
\hline $\begin{array}{l}\text { Composite primary efficacy } \\
\text { outcome }\end{array}$ & $78(2.7)$ & $82(2.7)$ & $0.97(0.71-1.31)$ & 0.003 & $131(4.4)$ & $175(5.7)$ & $0.77(0.62-0.96)$ & 0.02 \\
\hline Asymptomatic proximal DVT & $71(2.4)$ & $71(2.4)$ & - & - & $103(3.5)$ & $133(4.4)$ & - & - \\
\hline $\begin{array}{l}\text { Symptomatic proximal or } \\
\text { distal DVT }\end{array}$ & $7(0.2)$ & $6(0.2)$ & - & - & $13(0.4)$ & $15(0.5)$ & - & - \\
\hline $\begin{array}{l}\text { Symptomatic nonfatal } \\
\text { pulmonary embolism }\end{array}$ & $6(0.2)$ & $2(<0.1)$ & - & - & $10(0.3)$ & $14(0.5)$ & - & - \\
\hline VTE-related death & $3(0.1)$ & $6(0.2)$ & - & - & $19(0.6)$ & $30(1.0)$ & - & - \\
\hline
\end{tabular}

* Two-sided $95 \%$ confidence intervals $(\mathrm{Cl})$ for weighted relative risks were calculated with the use of asymptotic methods, with weights based on sample sizes per stratum of geographic region.

$\dagger$ The $P$ values were calculated on the basis of the normal approximation. The $P$ value for the day 10 analysis is a one-sided $P$ value for noninferiority, calculated in the per-protocol population; the $\mathrm{P}$ value for the day 35 analysis is a two-sided $\mathrm{P}$ value for superiority, calculated in the modified intention-to-treat population.

patients (2.8\%) who were receiving rivaroxaban as compared with 49 of 4001 patients (1.2\%) who were receiving enoxaparin (relative risk, 2.3; 95\% CI, 1.63 to 3.17; $\mathrm{P}<0.001$ ), and fatal bleeding occurred in 5 patients in the rivaroxaban group and in 1 patient in the enoxaparin group. Between day 1 and day 35, an episode of clinically relevant bleeding occurred in 164 of 3997 patients (4.1\%) in the group that received extended-duration rivaroxaban as compared with 67 of 4001 patients $(1.7 \%)$ in the group that received enoxaparin followed by placebo (relative risk, 2.5; 95\% CI, 1.85 to $3.25 ; \mathrm{P}<0.001)$. There was no evidence of heterogeneity according to subgroup with respect to clinically relevant bleeding at day 35 (Fig. S2 in the Supplementary Appendix). Fatal bleeding occurred in 7 patients in the group that received extended-duration rivaroxaban and in 1 patient in the group that received enoxaparin followed by placebo. The seven fatal bleeding events involved pulmonary bleeding (in 3 patients), intracranial bleeding (in 2 patients), and retroperitoneal and gastrointestinal bleeding (each in 1 patient). In the enoxaparin group there was one death due to tracheal bleeding.

The adverse-event profiles and the incidence of any cardiovascular event were similar up to day 35 in the group that received extended-duration rivaroxaban and the group that received enoxa- parin followed by placebo (Table 4). Over the total study period, alanine aminotransferase elevations greater than three times the upper limit of the normal range with a concurrent elevation in the bilirubin level that was greater than two times the upper limit of the normal range occurred in 7 of 3364 patients $(0.2 \%)$ in the rivaroxaban group and 7 of 3382 patients $(0.2 \%)$ in the enoxaparin group (measurements were not available for approximately $16 \%$ of the patients). The incidence of death from any cause over the entire study period was similar in the two groups.

\section{NET CLINICAL BENEFIT OR HARM}

By day 10, an event of the primary efficacy outcome or major or clinically relevant nonmajor bleeding (the measure of net clinical benefit or harm) had occurred in 216 of 3266 patients (6.6\%) in the rivaroxaban group, as compared with 151 of 3291 patients (4.6\%) in the enoxaparin group (relative risk, 1.44; 95\% CI, 1.18 to 1.77; $\mathrm{P}<0.001$ ) (Table 3). By day 35, an event of this composite outcome had occurred in 286 of 3042 patients $(9.4 \%)$ in the group that received extended-duration rivaroxaban, as compared with 240 of 3082 patients $(7.8 \%)$ in the group that received enoxaparin followed by placebo (relative risk, 1.21; 95\% CI, 1.03 to 1.43; $\mathrm{P}=0.02$ ) (Table 3). 


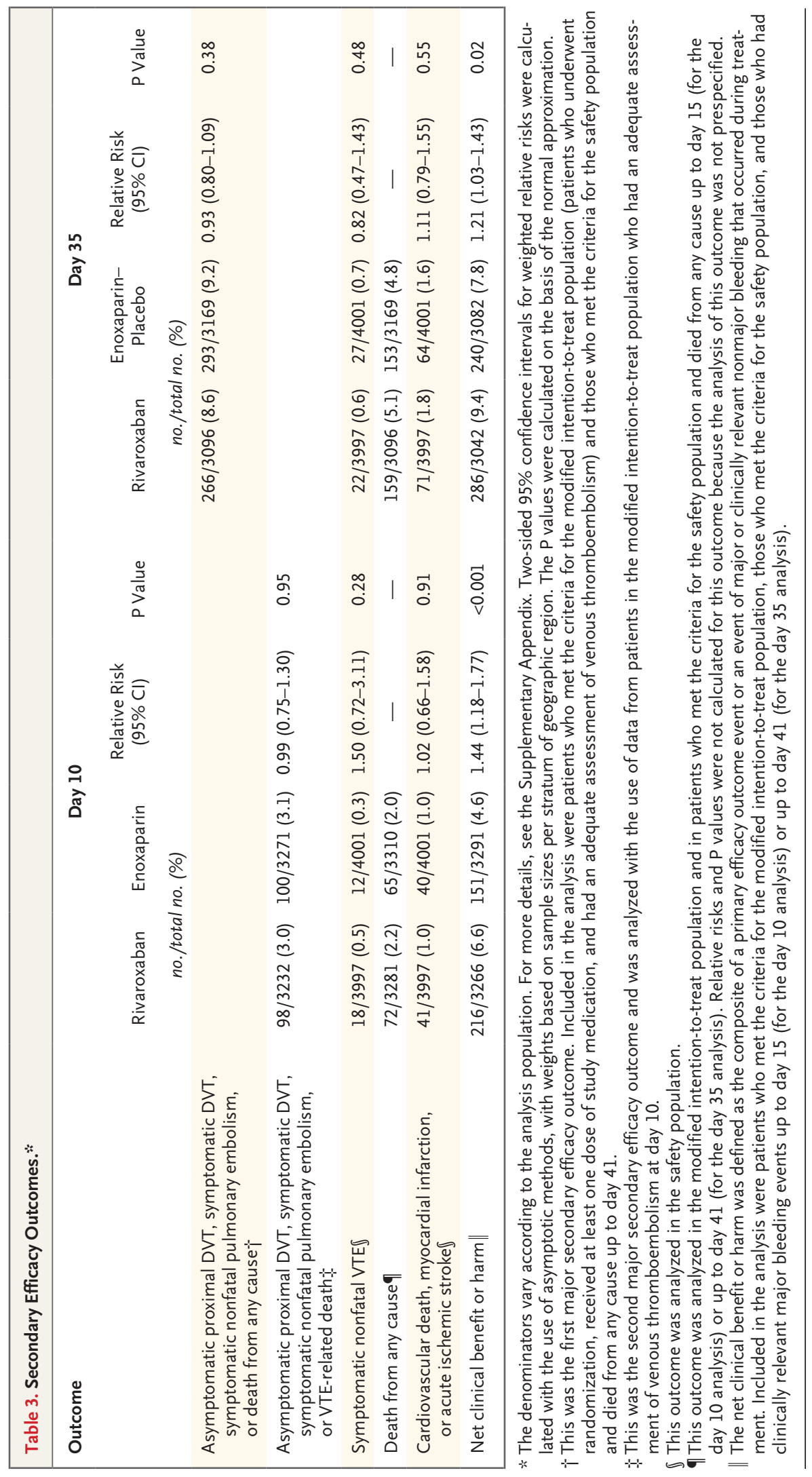




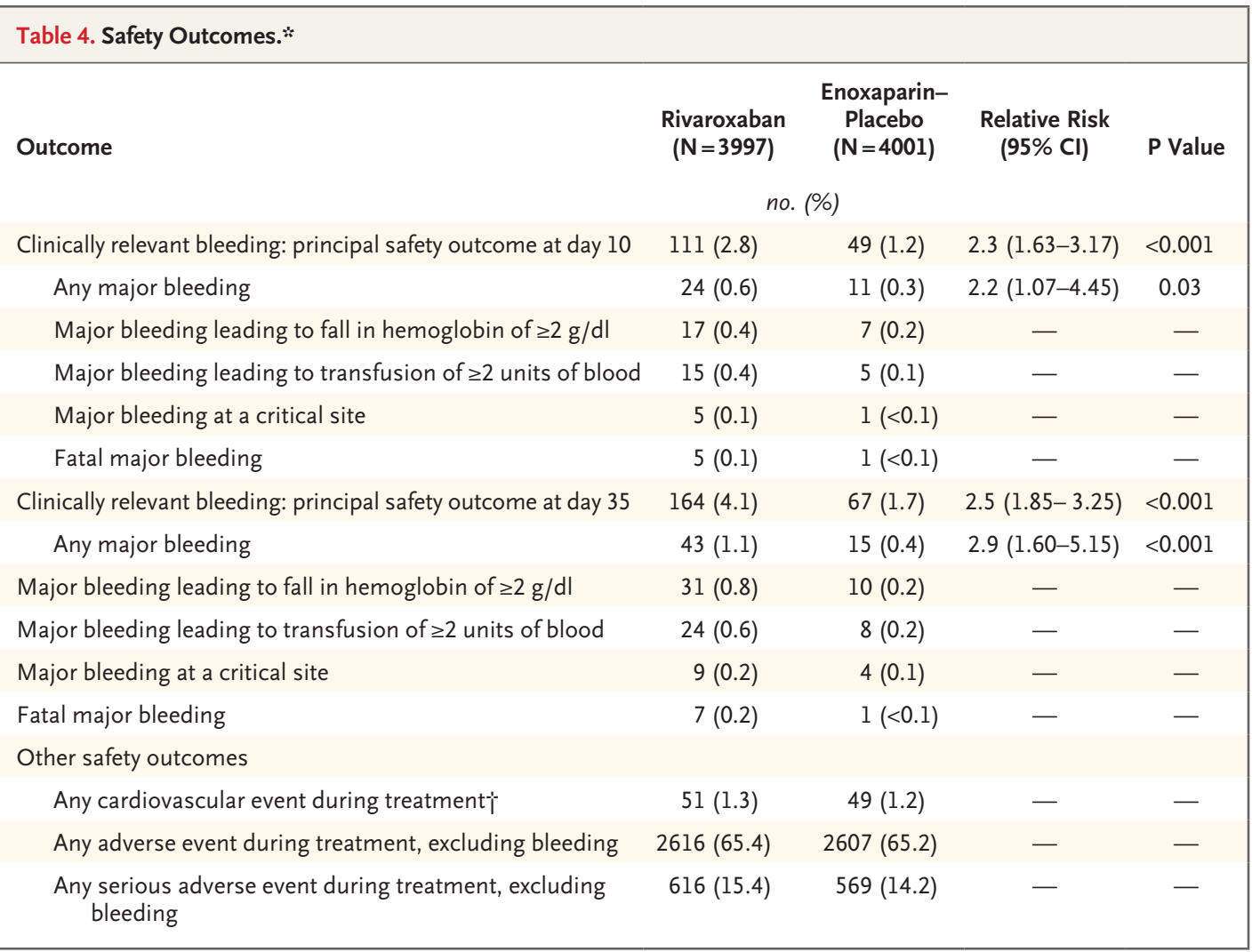

* Two-sided $95 \%$ confidence intervals for weighted relative risks were calculated with the use of asymptotic methods, with weights based on sample sizes per stratum of geographic region. The $P$ values were calculated on the basis of the normal approximation. Outcomes for which relative risks and $P$ values are not shown are those for which the analyses were not prespecified.

$\dagger$ Included are events of cardiovascular death, ischemic stroke, acute myocardial infarction, and acute stroke of unknown type.

\section{DISCUSSION}

In MAGELLAN, we investigated the efficacy and safety of rivaroxaban for the prevention of venous thromboembolism in acutely ill hospitalized patients. The study included a population of patients that was heterogeneous with respect to the medical condition leading to hospitalization and the geographic origin and race of the patients. ${ }^{13}$ We found that for the standard duration of therapy (10 \pm 4 days), rivaroxaban was noninferior to enoxaparin. Rivaroxaban administered for an extended duration (35 \pm 4 days) was superior to enoxaparin administered for the standard duration (10 \pm 4 days) followed by placebo.

The rate of clinically relevant bleeding (a composite of major or nonmajor bleeding) was significantly higher in the rivaroxaban group than in the enoxaparin group. The incidence of major bleeding events was also significantly higher in the rivaroxaban group than in the enoxaparin group, with the majority of events in both groups leading to a fall in the hemoglobin level of at least $2 \mathrm{~g}$ per deciliter or to the transfusion of at least 2 units of blood. A reduction in the rate of death related to venous thromboembolism and an increase in the rate of death related to bleeding were seen with extended-duration rivaroxaban prophylaxis. However, there was no reduction with rivaroxaban in the rate of death from any cause, and the incidence was similar in the two groups - findings that were consistent with those in other studies. ${ }^{11,12}$ The prespecified analysis of net clinical benefit or harm did not show a benefit with rivaroxaban at either day 10 or day 35 .

Factors that influence the risk of bleeding in acutely ill medical patients are not well defined. A recent study identified risk factors at admission that were associated with in-hospital bleeding in acutely ill medical patients; the risk factors included active gastroduodenal ulcer, prior bleeding, low platelet count, increasing age, hepatic 
or renal failure, the presence of a central venous catheter, rheumatic disease, and cancer. ${ }^{15}$ The median age of the patients in MAGELLAN was 71 years, and approximately $20 \%$ had impaired renal function (creatinine clearance less than $50 \mathrm{ml}$ per minute). In addition, just over $7 \%$ had active cancer ( $17 \%$ had a history of cancer), and approximately $4 \%$ had an acute inflammatory or rheumatic disease. There were no differences in characteristics between patients in the rivaroxaban group and those in the enoxaparin group that could explain the disparity in bleeding rates at day 10. However, the factors listed above, in combination with the acute medical illness, could have contributed to the increased bleeding risk with extended thromboprophylaxis that was observed in these patients as compared with patients undergoing major orthopedic surgery - a generally younger and healthier patient population - among whom the benefits and safety of extended thromboprophylaxis have been well established. ${ }^{9}$ The results of the current study support those from other studies that showed the efficacy of extended thromboprophylaxis ${ }^{11}$ but also the increased risk of bleeding. ${ }^{11,12}$

One limitation of our trial was the inclusion of asymptomatic proximal deep-vein thrombosis, as detected on ultrasonography, as part of the primary efficacy outcome. Ultrasonography is not performed routinely in medical patients. ${ }^{12}$ The use of this test may have influenced the trial in two ways. First, the performance of ultrasonography at day 10 may have influenced the subsequent natural history of the disease because it may have resulted in the treatment of asymptomatic disease. This could account for the risk reduction at day 35 that was lower than anticipated. In an analysis of the primary efficacy outcome events between day 11 and day 35, the relative risk reduction with extended-duration rivaroxaban was $35 \%$ (data not shown), which is closer to the expected $40 \%$. Second, a substantial subgroup of patients who underwent randomization could not be evaluated for the primary outcome because they did not undergo ultraso- nography or because their ultrasonograms could not be properly assessed. The extent to which the lack of data for these patients may have influenced the findings of the trial is not clear.

In conclusion, we compared enoxaparin, at a dose of $40 \mathrm{mg}$ once daily for 10 days, with rivaroxaban, at a dose of $10 \mathrm{mg}$ once daily for 35 days, for thromboprophylaxis in patients hospitalized with acute medical illness. The efficacy of standard-duration rivaroxaban was similar to that of enoxaparin, whereas the efficacy of extendedduration rivaroxaban was superior to that of enoxaparin. However, rivaroxaban was associated with an increased risk of clinically relevant bleeding.

Dr. Cohen reports serving on advisory boards for Bayer, BristolMyers Squibb, Daiichi Sankyo, Johnson \& Johnson, Pfizer, Portola, and Sanofi and receiving consulting fees, lecture fees, payment for manuscript preparation, and payment for the development of educational presentations from Astellas, AstraZeneca, Bayer, Boehringer Ingelheim, Bristol-Myers Squibb, Daiichi Sankyo, GlaxoSmithKline, Johnson \& Johnson, Mitsubishi Pharma, Pfizer, Portola, Sanofi, Schering Plough, and Takeda; Dr. Spiro, being an employee of, holding stock options in, and receiving travel support from Bayer; Dr. Büller, serving on advisory boards for and receiving consulting fees and payment for manuscript preparation through his institution from Bayer and Daiichi Sankyo; Dr. Haskell, being an employee of and holding stock options in Johnson \& Johnson; Dr. Hull, receiving consulting fees from LEO Pharma, Bayer, Portola, and Sanofi and lecture fees, grant support, and reimbursement for travel expenses from LEO Pharma and Sanofi; Dr. Mebazaa, serving on advisory boards for Cardiorentis and the Medicines Company, and receiving consulting fees from Pronota, lecture fees from Alere, Edwards, Orion, and Thermo Fisher, and royalties from Pronota; Dr. Merli, receiving consulting fees from Bristol-Myers Squibb and grant support through his institution from Bristol-Myers Squibb and Sanofi-Aventis, Dr. Schellong, serving on advisory boards for Bayer, Boehringer Ingelheim, and Novartis, and receiving consulting fees from Bayer, Boehringer Ingelheim, Daiichi Sankyo, GlaxoSmithKline, Novartis, and Sanofi Aventis, lecture fees from Bayer, Bristol-Myers Squibb, Boehringer Ingelheim, Daiichi Sankyo, GlaxoSmithKline, LEO Pharma, and Sanofi Aventis and payment for the development of educational presentations from Daiichi Sankyo and Bayer; Dr. Spyropoulos, receiving consulting fees from Astellas, Boehringer Ingelheim, Bristol-Myers Squibb, and Johnson \& Johnson; and Dr. Tapson, receiving consulting fees from Bayer, Bristol-Myers Squibb, Covidien, and Sanofi, lecture fees from Covidien and Sanofi, payment for the development of educational presentations from Sanofi, and grant support through his institution from Bayer and Sanofi. No other potential conflict of interest was reported.

Disclosure forms provided by the authors are available with the full text of this article at NEJM.org.

REFERENCES

1. Cohen AT, Alikhan R, Arcelus JI, et al. Assessment of venous thromboembolism risk and the benefits of thromboprophylaxis in medical patients. Thromb Haemost 2005;94:750-9.

2. Samama MM. An epidemiologic study of risk factors for deep vein thrombosis in medical outpatients: the Sirius study. Arch Intern Med 2000;160:3415-20. 3. Alikhan R, Cohen AT, Combe S, et al. Risk factors for venous thromboembolism in hospitalized patients with acute medical illness: analysis of the MEDENOX Study. Arch Intern Med 2004;164:963-8. 4. Dentali F, Douketis JD, Gianni M, Lim W, Crowther MA. Meta-analysis: anticoagulant prophylaxis to prevent symptomatic venous thromboembolism in hospi- 
talized medical patients. Ann Intern Med 2007;146:278-88.

5. Samama MM, Cohen AT, Darmon JY, et al. A comparison of enoxaparin with placebo for the prevention of venous thromboembolism in acutely ill medica patients. N Engl J Med 1999;341:793-800. 6. Fraisse F, Holzapfel L, Couland JM, et al. Nadroparin in the prevention of deep vein thrombosis in acute decompensated COPD. Am J Respir Crit Care Med 2000; 161:1109-14.

7. Leizorovicz A, Cohen AT, Turpie AGG, Olsson CG, Vaitkus PT, Goldhabe SZ. Randomized, placebo-controlled tria of dalteparin for the prevention of venous thromboembolism in acutely ill medical patients. Circulation 2004;110:874-9.

8. Cohen AT, Davidson BL, Gallus AS, et al. Efficacy and safety of fondaparinux for the prevention of venous thromboembolism in older acute medical patients: randomised placebo controlled trial. BMJ 2006;332:325-9.

9. Geerts WH, Bergqvist D, Pineo GF, et al. Prevention of venous thromboembolism: American College of Chest Physicians Evidence-Based Clinical Practice Guidelines (8th Edition). Chest 2008;133: Suppl:381S-453S.

10. Spencer FA, Lessard D, Emery C, Reed G, Goldberg RJ. Venous thromboembolism in the outpatient setting. Arch Intern Med 2007;167:1471-5.

11. Hull RD, Schellong SM, Tapson VF, et al. Extended-duration venous thromboembolism prophylaxis in acutely ill medical patients with recently reduced mobility: a randomized trial. Ann Intern Med 2010;153:8-18.
12. Goldhaber SZ, Leizorovicz A, Kakkar AK, et al. Apixaban versus enoxaparin for thromboprophylaxis in medically ill patients. N Engl J Med 2011;365:2167-77.

13. Cohen AT, Spiro TE, Büller HR, et al. Extended-duration rivaroxaban thromboprophylaxis in acutely ill medical patients: MAGELLAN study protocol. J Thromb Thrombolysis 2011;31:407-16.

14. Hochberg Y, Hommel G. Simes' test of multiple hypotheses. In: Kotz S, ed. Encyclopedia of statistical sciences, update volume 2. New York: Wiley, 1998:418-22. 15. Decousus H, Tapson VF, Bergmann JF, et al. Factors at admission associated with bleeding risk in medical patients: findings from the IMPROVE investigators. Chest 2011;139:69-79.

Copyright (c) 2013 Massachusetts Medical Society.

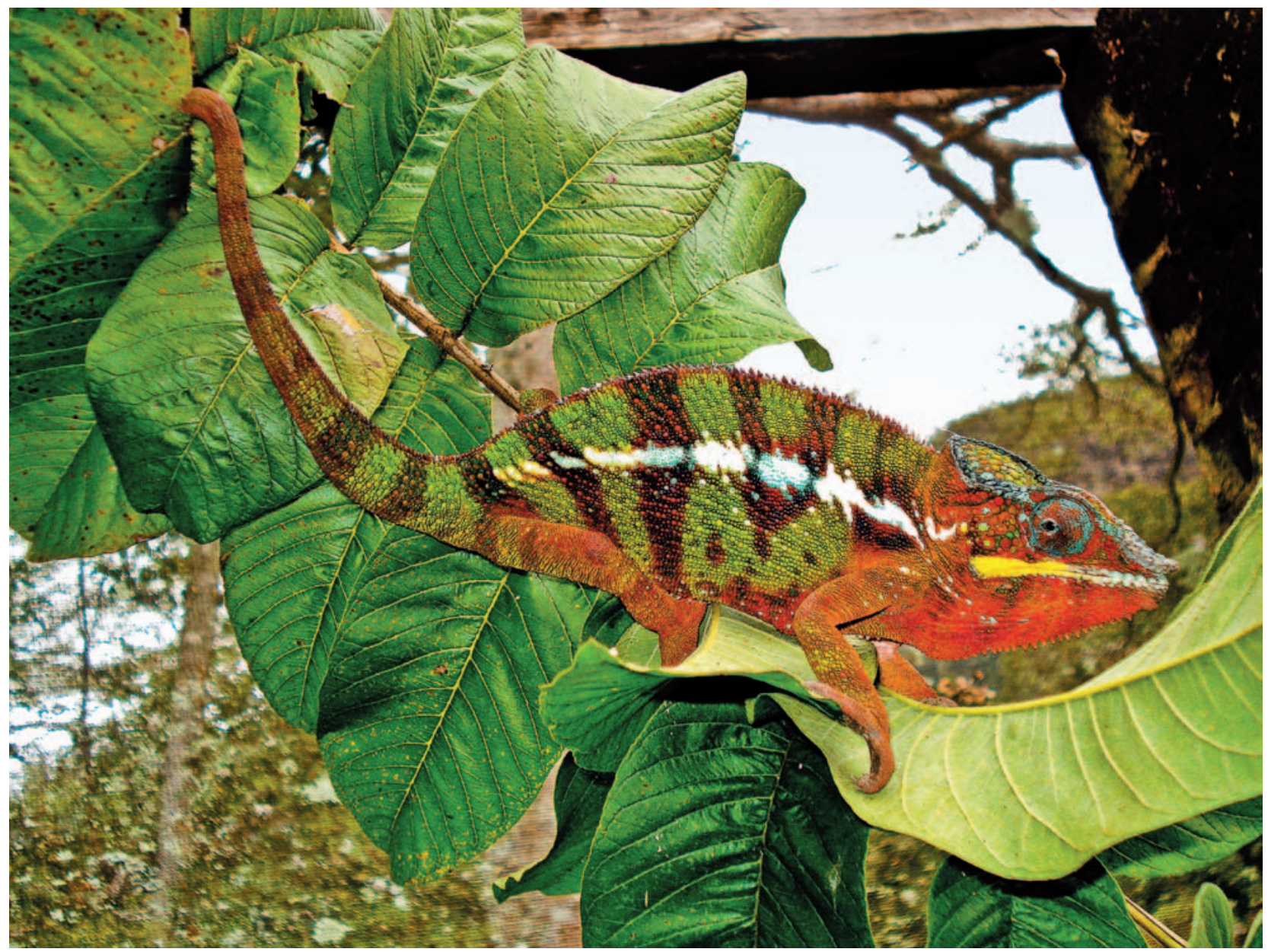

Panther Chameleon, Madagascar

Philip Cokkinos, M.D. 